\title{
Benefit of Cisplatin With Definitive Radiotherapy in Older Women With Cervical Cancer
}

\author{
Michael Xiang, MD, PhD, and Elizabeth A. Kidd, MD
}

\begin{abstract}
Background: Cisplatin with definitive radiotherapy (RT) is considered the standard of care for cervical cancer; however, older women are frequently undertreated and have worse outcomes compared with younger patients. Because women aged $\geq 65$ years have been disproportionately underrepresented in clinical trials, uncertainties exist regarding how much they benefit from the addition of cisplatin to RT. Patients and Methods: Women aged $\geq 65$ years with nonmetastatic cervical cancer treated with definitive external-beam RT and brachytherapy were identified in the SEER-Medicare database. Death attributable to cervical cancer (cancer-specific mortality [CSM]) was evaluated against competing risks of death using Gray's test. Propensity score analysis and the Fine-Gray multivariable regression model were used to adjust for baseline differences, including comorbidity. Results: The total cohort comprised 826 patients, of whom 531 (64\%) received cisplatin, 233 (28\%) were FIGO stage I, 374 (45\%) were stage II, and 219 (27\%) were stage III-IVA. Older age and chronic kidney disease significantly predicted omission of cisplatin. Virtually all cisplatin dosing was weekly, with a median of 5 cycles. Death from cervical cancer was significantly lower with cisplatin than without (5-year CSM, 31\% vs 39\%; $P=.02$; adjusted hazard ratio, 0.72 ; $P=.02$ ), which persisted in propensity score analysis. Receiving $\geq 5$ cycles was required for benefit, as no difference in CSM was seen in patients receiving 1 to 4 cycles versus no cisplatin. Subgroup analyses revealed that the benefit of cisplatin persisted in women aged $\geq 75$ years and those with early-stage disease. Incidence of cytopenia, nausea/vomiting, and hypovolemia increased in patients treated with cisplatin. Conclusions: Administration of cisplatin with definitive RT in women aged $\geq 65$ years was associated with a significant benefit in the incidence of death attributable to cervical cancer, despite competing risks for mortality in an older population. Receiving at least 5 cycles of weekly cisplatin was required for benefit.
\end{abstract}

J Natl Compr Canc Netw 2019;17(8):969-975 doi: $10.6004 /$ jnccn.2019.7289

Department of Radiation Oncology, Stanford University, Stanford, California.

\section{Background}

Cervical cancer is commonly viewed as a disease affecting younger women. However, age at diagnosis follows a bimodal distribution, with peaks at 30 to 39 years and 60 to 69 years. ${ }^{1}$ When hysterectomy is taken into account, the highest incidence of cervical cancer is in women aged $>65$ years. $^{2,3}$ Moreover, as the population ages and a younger generation of women benefit from vaccination against HPV, the burden of cervical cancer in older women is expected to increase further. ${ }^{1,4}$

The optimal treatment of older women with cervical cancer is unclear. In the United States, women aged $\geq 65$ years account for $25 \%$ of cervical cancer cases but $40 \%$ of deaths from cervical cancer. ${ }^{5}$ Older women tend to present with more advanced disease, ${ }^{6-8}$ yet are frequently undertreated $^{7-10}$ and have worse outcomes than younger women, ${ }^{1,5,8}$ indicating a clear need for additional research aimed at addressing age-related disparities in treatment and outcomes. RTOG 90-01, ${ }^{11}$ other clinical trials, ${ }^{12}$ and a meta-analysis ${ }^{13}$ have shown that concurrent chemoradiation (CRT) increases survival compared with definitive radiotherapy (RT) alone, but the fraction of patients aged $>65$ years was only $6 \%$ to $7 \%{ }^{13}$ This finding precludes drawing robust conclusions about outcomes in older women from randomized trials.

Cisplatin is the preferred chemotherapeutic agent in patients with cervical cancer undergoing definitive CRT. ${ }^{14}$ However, the added benefit of cisplatin in elderly women is unclear because of concern for increased toxicity and decreased efficacy, and prior studies have yielded conflicting findings. ${ }^{15,16}$ Furthermore, older patients are prone to competing risks of death, which may diminish the survival benefit from more intensive cancer treatment. Given these uncertainties, we analyzed patterns of care and cancer-specific outcomes for women aged $\geq 65$ years treated with definitive CRT \pm cisplatin. The SEERMedicare database was used given its large sample size, national representation of real-life practices and

See JNCCN.org for supplemental online content. 
outcomes, cause of death information, and availability of chemotherapy, RT, and comorbidity data.

\section{Patients and Methods}

\section{Data Source}

The SEER registry captures all incident cancers from 17 regional registries corresponding to $30 \%$ of the US population. The SEER-Medicare database links patients in SEER with their Medicare claims, which allows for determination of specific diagnoses and procedures across time using ICD and Healthcare Common Procedure Coding System (HCPCS) codes. ${ }^{17}$ All data were deidentified. This study was approved by the Stanford University Institutional Review Board.

\section{Cohort Identification}

We queried the 2016 linkage of the SEER-Medicare database to identify women aged $\geq 65$ years with nonmetastatic, microscopically confirmed invasive cervical squamous cell carcinoma or adenocarcinoma. Cases were included from 2004 and onward, when modern staging information became available in SEER. Patients with unknown FIGO stage or nodal stage were excluded. To ensure adequate capture of claims and to analyze a more uniform population, patients were required to be enrolled in Medicare Parts A and $\mathrm{B}$, have no HMO coverage within 12 months of diagnosis, and be enrolled in Medicare for age only.

All patients received definitive RT, defined as having Medicare claims for both external-beam RT and brachytherapy (codes listed in supplemental eTable 1, available with this article at JNCCN.org) within 6 months after diagnosis, and no hysterectomy according to the SEER sitespecific surgery field. Receipt of cisplatin was determined by claims within 6 months after diagnosis with HCPCS codes J9060, J9062, C9418. Figure 1 summarizes the schema used to identify the study cohort.

\section{Determination of Study Variables and Outcomes}

Comorbidity was calculated using the Charlson comorbidity index as previously described. ${ }^{17}$ Presence of chronic kidney disease was determined from claims before diagnosis, and acute toxicities were inferred from claims within 9 months after diagnosis (supplemental eTable 2), as in prior claimsbased analyses. ${ }^{17,18}$ The number of cisplatin cycles was determined as the number of cisplatin claims within 90 days of the first cisplatin claim. All other study variables were obtained directly from the SEER-Medicare database. The primary outcome was death attributable to cervical cancer (cancer-specific mortality [CSM]) determined by the SEER cause-specific death classification and the SEER cause of death to site recode field (code 27010). CSM was chosen as the primary outcome, because healthier patients may be more likely to tolerate and receive cisplatin, which would confound overall survival. ${ }^{17,19}$ For the 2016 SEER-Medicare linkage, patient follow-up was through December 2014.

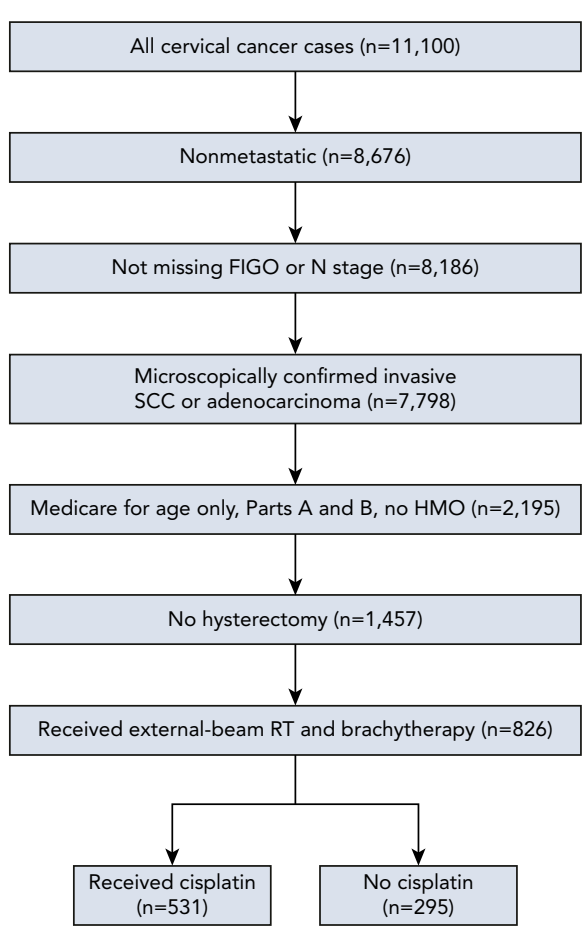

Figure 1. Cohort identification algorithm.

Abbreviations: RT, radiotherapy; SCC, squamous cell carcinoma.

\section{Statistical Analysis}

Baseline characteristics were compared using the chisquare or Wilcoxon rank-sum test. Multivariable logistic regression was used to identify predictors for receiving cisplatin versus no cisplatin. The cumulative incidence of death from cervical cancer (CSM) was estimated in the presence of other-cause mortality as a competing risk and compared using Gray's test. ${ }^{17}$ Propensity score analysis was performed with 1:1 matching as previously described. ${ }^{17}$ Briefly, propensity scores corresponding to the likelihood of treatment with cisplatin were estimated with logistic regression using the same variables as in the multivariable analysis. Patients in the cisplatin cohort were then matched to those in the noncisplatin cohort using a caliper width of 0.2 times the standard deviation of the logit of the propensity scores, generating matched cohorts with balanced baseline characteristics. For multivariable analysis, the proportional hazards model of Fine and Gray was used to estimate adjusted hazard ratios (aHRs) for CSM and acute toxicity. MATLAB 9.4 (MathWorks) and $\mathrm{R}$ version 3.3.3 (R Foundation for Statistical Computing) were used for calculations. All statistical tests were 2-sided and considered significant at $P<.05$.

\section{Results}

A total of 826 patients underwent definitive RT, of whom $531(64 \%)$ received cisplatin and 295 (36\%) did not. Overall, 711 (86\%) were node-negative, 233 (28\%) were FIGO 
Table 1. Patient Characteristics and Predictors for Receiving Cisplatin

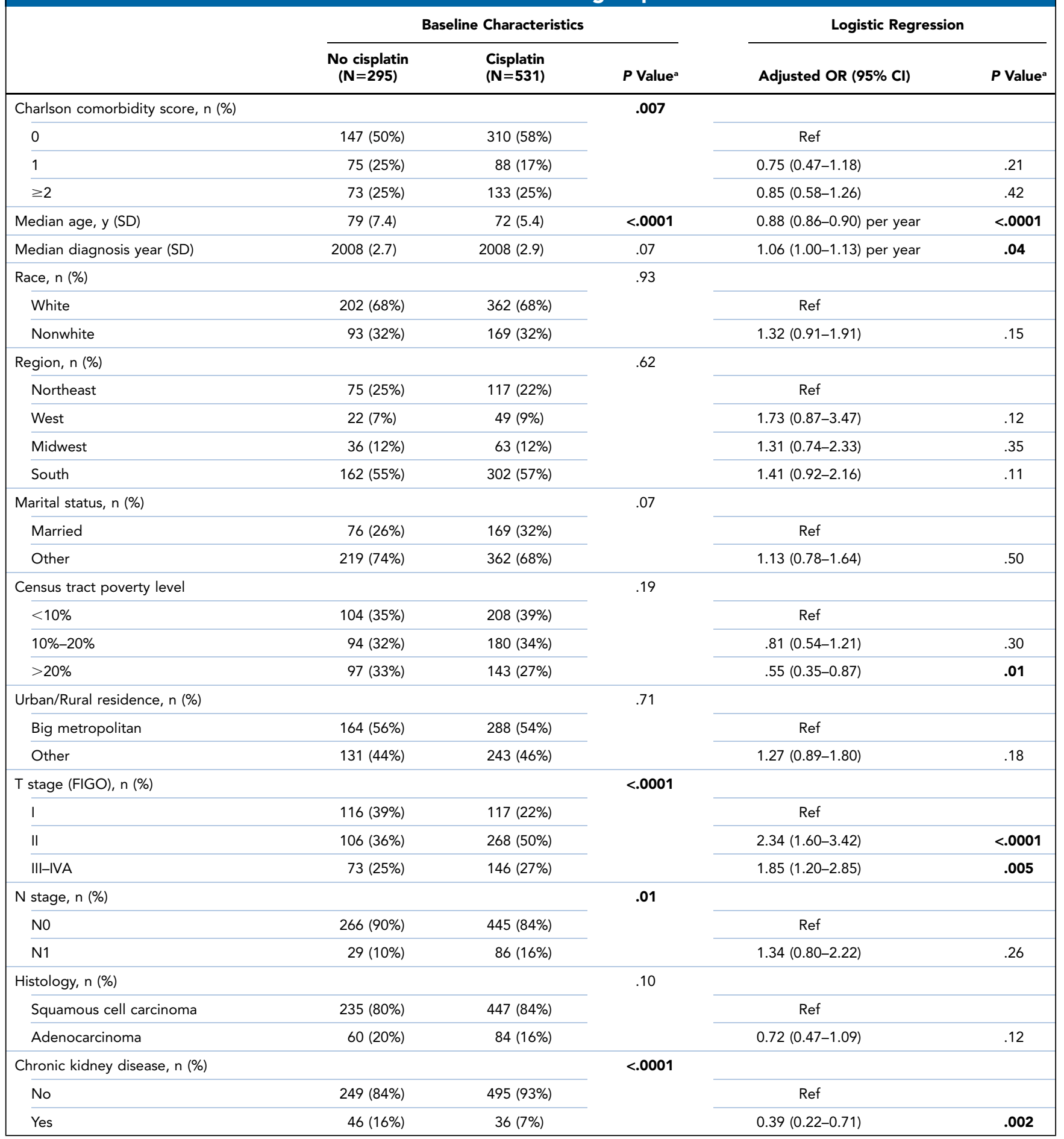

Abbreviation: OR, odds ratio.

aBolded values are statistically significant $(P<.05)$.

stage I, 374 (45\%) were stage II, and 219 (27\%) were stage III-IVA. Median follow-up was 4.5 years in living patients.

Table 1 lists the baseline characteristics of the study patients. Those treated with cisplatin tended to be younger, were more likely to have FIGO stage II or node-positive disease, and were less likely to have chronic kidney disease. In multivariable logistic regression, more advanced FIGO stage and more recent year of diagnosis 
predicted treatment with cisplatin, whereas older age, high census tract poverty level, and chronic kidney disease predicted omission of cisplatin.

Death from cervical cancer (CSM) was significantly reduced for patients treated with cisplatin versus without (5-year CSM, 31\% vs 39\%; $P=.02$; Figure 2). Propensity score analysis (432 total patients; 216 per matched cohort) resulted in groups that were well-balanced (supplemental eTable 3) and yielded similar findings favoring treatment with cisplatin (5-year CSM, $31 \%$ vs $41 \%$; $P=.046$; supplemental eFigure 1 ).

In the cisplatin cohort, virtually all patients received weekly administration, and the median number of cycles was 5 (supplemental eFigure 2). Survival benefit was superior in patients receiving $\geq 5$ cycles of cisplatin $(n=351)$ versus 1 to 4 cycles ( $\mathrm{n}=180$ ) (5-year CSM, $28 \%$ vs $38 \%$; $P=.01)$. By contrast, there was no significant difference between 0 cycles (no cisplatin) and 1 to 4 cycles of cisplatin (5-year CSM, $39 \%$ vs $38 \%$; $P=.83$; Figure 3 ).

In subgroup analyses, the benefit of cisplatin persisted in women aged $\geq 75$ years $(n=381)$, those with nodenegative disease $(n=711)$, or both $(n=335)$ (supplemental eFigures 3-5). Furthermore, cisplatin benefited patients with node-negative FIGO stage I $(n=211)$ or II disease $(\mathrm{n}=325)$ (supplemental eFigures 6 and 7). In each case, $\geq 5$ cycles of cisplatin was required for benefit, whereas receiving 1 to 4 cycles was not significantly different from 0 cycles. In women at lowest risk for recurrence (FIGO stage I-IIA, node-negative, and tumor $<4 \mathrm{~cm}$ ), no benefit was seen with cisplatin $(n=125$; supplemental eFigure 8).

On multivariable analysis, the aHR for CSM corresponding to the administration of cisplatin was 0.72 (95\% CI, 0.54-0.96; $P=.02$; Table 2). When the number of cisplatin cycles was categorized as 0,1 to 4 , or $\geq 5$, the aHR for receiving 1 to 4 cycles was 0.91 (95\% CI, $0.65-1.27 ; P=.57$ ) and the aHR for receiving $\geq 5$ cycles

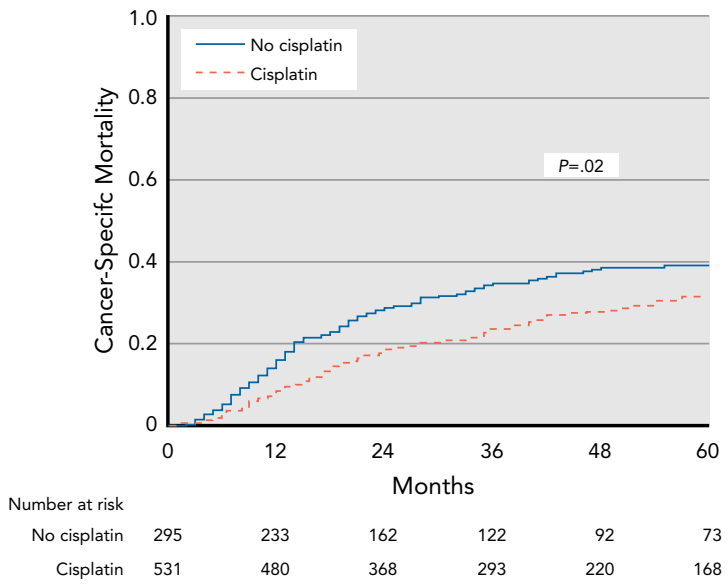

Figure 2. Cumulative incidence of death from cervical cancer according to receipt of cisplatin. was 0.62 (95\% CI, 0.46-0.85; $P=.003$ ). Other significant predictors of CSM included age, diagnosis year, FIGO stage, and lymph node involvement (Table 2).

Lastly, we analyzed the incidence of acute toxicities. Receiving cisplatin was not associated with an increase in gastrointestinal (diarrhea) (aHR, 1.04; $P=.78$ ) or genitourinary (cystitis/dysuria) toxicity (aHR, 1.13; $P=.48$ ). However, cisplatin was associated with increased hematologic (cytopenia) toxicity (aHR, 2.71; 95\% CI, $1.81-4.08 ; P<.0001$ ), nausea/vomiting (aHR, 2.12; 95\% CI, 1.57-2.86; $P<.0001$ ), and hypovolemia (aHR, 1.73; $95 \%$ CI, 1.28-2.35; $P=.0004)$.

\section{Discussion}

In this large national cohort of women aged $\geq 65$ years with cervical cancer treated with definitive RT, cisplatin administration was associated with an $8 \%$ to $10 \%$ absolute decrement in death from cervical cancer at 5 years, despite cisplatin recipients having more advanced and/ or node-positive disease. This finding persisted in the propensity score and multivariable analyses.

Notably, our results are similar to those of randomized studies comprising younger women. A meta-analysis of 13 randomized trials found that CRT confers a $6 \%$ absolute survival advantage at 5 years compared with RT alone. ${ }^{13}$ RTOG 90-01 reported a survival advantage of approximately $20 \%$ for high-dose cisplatin +5 -FU delivered with RT. ${ }^{11,20}$ Median age in both the metaanalysis and RTOG 90-01 was 47 years.

Additionally, we found that cisplatin benefited all subgroups, even those ostensibly with lower-risk disease. The only subgroup that did not show a benefit with cisplatin was women at lowest risk for recurrence (nonbulky, node-negative, early-stage disease [FIGO I-IIA]); however, these patients would also not have qualified for enrollment in RTOG 90-01.

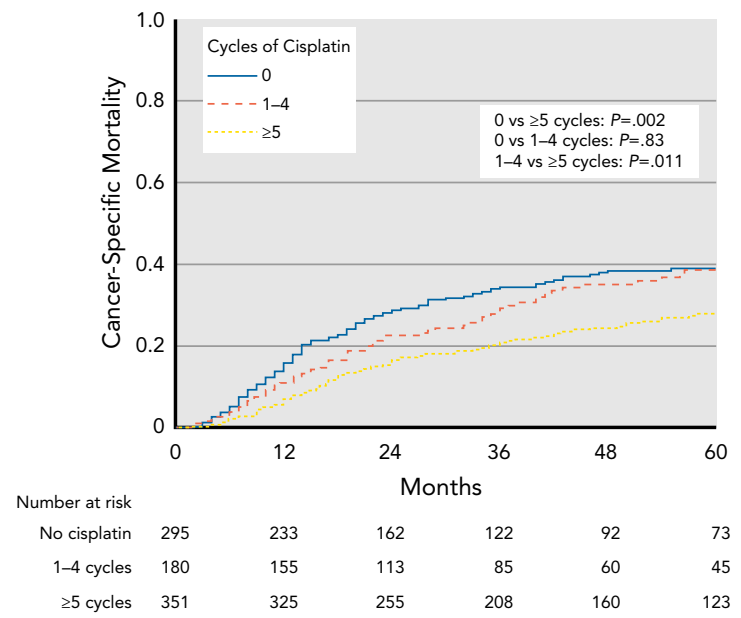

Figure 3. Cumulative incidence of death from cervical cancer according to cycles of cisplatin. 
Table 2. Predictors of Death From Cervical Cancer on Multivariable Analysis

\begin{tabular}{|c|c|c|}
\hline & Adjusted HR $(95 \% \mathrm{Cl})$ & $P$ Value \\
\hline \multicolumn{3}{|l|}{ Receipt of cisplatin } \\
\hline No & Ref & \\
\hline Yes & $0.72(0.54-0.96)$ & .02 \\
\hline \multicolumn{3}{|l|}{ Charlson comorbidity score } \\
\hline 0 & Ref & \\
\hline 1 & $0.80(0.53-1.20)$ & .28 \\
\hline$\geq 2$ & $1.13(0.84-1.51)$ & .42 \\
\hline Age & $1.02(1.00-1.04)$ per year & .02 \\
\hline Diagnosis year & $0.92(0.88-0.96)$ per year & .0004 \\
\hline \multicolumn{3}{|l|}{ Race } \\
\hline White & Ref & \\
\hline Nonwhite & $0.79(0.58-1.07)$ & .12 \\
\hline \multicolumn{3}{|l|}{ Region } \\
\hline Northeast & Ref & \\
\hline West & $1.13(0.66-1.93)$ & .66 \\
\hline Midwest & $0.87(0.56-1.36)$ & .55 \\
\hline South & $1.21(0.86-1.70)$ & .27 \\
\hline \multicolumn{3}{|l|}{ Marital status } \\
\hline Married & Ref & \\
\hline Other & $0.95(0.73-1.24)$ & .71 \\
\hline \multicolumn{3}{|l|}{ Census tract poverty level } \\
\hline$<10 \%$ & Ref & \\
\hline $10 \%-20 \%$ & $1.14(0.85-1.52)$ & .38 \\
\hline$>20 \%$ & $0.95(0.67-1.33)$ & .76 \\
\hline \multicolumn{3}{|l|}{ Urban/Rural residence } \\
\hline Big metropolitan & Ref & \\
\hline Other & $1.16(0.89-1.52)$ & .27 \\
\hline \multicolumn{3}{|l|}{ T stage (FIGO) } \\
\hline I & Ref & \\
\hline ॥ & $1.84(1.32-2.55)$ & .0003 \\
\hline III-IVA & $2.65(1.85-3.79)$ & $<.0001$ \\
\hline \multicolumn{3}{|l|}{$\mathrm{N}$ stage } \\
\hline No & Ref & \\
\hline N1 & $1.66(1.20-2.30)$ & .002 \\
\hline \multicolumn{3}{|l|}{ Histology } \\
\hline Squamous cell carcinoma & Ref & \\
\hline Adenocarcinoma & $1.19(0.87-1.64)$ & .28 \\
\hline \multicolumn{3}{|l|}{ Chronic kidney disease } \\
\hline No & Ref & \\
\hline Yes & $1.16(0.69-1.98)$ & .58 \\
\hline
\end{tabular}

Abbreviation: $\mathrm{HR}$, hazard ratio.

aBolded values are statistically significant $(P<.05)$.
The added benefit of chemotherapy in older patients undergoing RT has been controversial because of concerns regarding increased toxicities, decreased effectiveness, and competing risks for death in the elderly. For example, CRT was not superior to RT alone in patients with head and neck cancer aged $\geq 65$ or $>70$ years $^{21,22}$; however, elderly patients with lung, ${ }^{23}$ bladder, ${ }^{24}$ or endometrial cancer ${ }^{25}$ have been shown to benefit from combined modality treatment. Despite the presence of competing risks for mortality in an older population, we found that the administration of cisplatin with definitive RT substantially decreased the risk of dying from cervical cancer. Thus, our data suggest that the threat of death from cervical cancer remains sufficiently high in women aged $\geq 65$ years that the added benefit of cisplatin seems to outweigh the potential risks.

Our study addresses a significant gap in the existing literature because older women have been disproportionately underrepresented in clinical trials. Women aged $\geq 65$ years account for $25 \%$ of cervical cancer diagnoses, ${ }^{5}$ but comprised only $6 \%$ to $7 \%$ of patients in the CRT metaanalysis, ${ }^{13}$ and those aged $\geq 70$ years comprised $5 \%$ of patients in a pooled analysis of $4 \mathrm{GOG}$ trials. ${ }^{1}$ Consequently, the major clinical trials in cervical cancer have not reported subgroup analyses for older patients. Similarly, prior retrospective series have also been limited by small sample sizes, leading to indeterminate and even contradictory conclusions. For example, a Korean series of 105 women aged $\geq 65$ years found no added benefit from chemotherapy (5-year cancer-specific survival [CSS], $69 \%$ vs $67 \%$ ), ${ }^{15}$ whereas a series from China with 73 women aged $\geq 70$ years reported significantly improved CSS ( $87 \%$ vs $57 \%$ at 3 years) when chemotherapy was administered with RT. ${ }^{16}$

Our results are particularly noteworthy given the significant age-related disparities in treatment and outcomes in cervical cancer. Older women are frequently undertreated $^{7-10}$ and have worse outcomes than younger patients, ${ }^{1,5,8}$ but it was unclear whether this was caused by undertreatment or because chemotherapy may cause more harm than benefit in older patients. Our study strongly suggests that older women benefit from combined modality treatment, as is the standard of care in younger women. This is consistent with the meta-analysis and pooled GOG experience, both of which found no interaction between age and chemotherapy effect, although the number of elderly patients was limited. ${ }^{1,13}$ Moreover, in the GOG analysis, age did not adversely impact completion of chemotherapy or RT or the rate of toxicity. ${ }^{1}$ Interestingly, we found that age but not comorbidity score was predictive of receiving cisplatin in the logistic model, which substantiates a prior report that physicians are less likely to discuss standard therapies with older women. ${ }^{10}$

Although RTOG 90-01 used high-dose cisplatin + 5-FU administered every 3 weeks, subsequent studies 
have shown that single-agent cisplatin administered weekly is less toxic and equally efficacious. ${ }^{26-28}$ Virtually all patients in the cisplatin cohort of our study received weekly cisplatin, with a median of 5 cycles. Obtaining $\geq 5$ cycles was required for benefit, with no difference in CSM seen in patients receiving 1 to 4 cycles versus no cisplatin. A limitation of our study is that pharmacologic dose information is not available in the SEER-Medicare database. However, the most common dose of weekly cisplatin is $40 \mathrm{mg} / \mathrm{m}^{2},{ }^{26,27}$ suggesting that a total cisplatin dose of at least $200 \mathrm{mg} / \mathrm{m}^{2}$ may be required for clinical benefit. This threshold is similar to that established in the GOG 109 study of patients treated with CRT in the adjuvant setting, in which at least $210 \mathrm{mg} / \mathrm{m}^{2}$ (3 cycles of high-dose cisplatin) was required for benefit. ${ }^{29} \mathrm{~A}$ similar threshold of at least $200 \mathrm{mg} / \mathrm{m}^{2}$ total cisplatin was found to benefit patients with squamous cell carcinoma of the head and neck undergoing definitive CRT. ${ }^{30}$

We found that acute genitourinary and gastrointestinal toxicities were not increased in the cisplatin cohort, whereas rates of cytopenia, nausea/vomiting, and hypovolemia were higher and consistent with clinical experience. Prior studies have shown that CRT increases hematologic and gastrointestinal toxicities, ${ }^{12,13,20,29}$ although the rate of hematologic toxicity is lower with single-agent weekly cisplatin compared with high-dose cisplatin +5 -FU..$^{27,28,31}$ Whether the rate of gastrointestinal toxicity is also lower with weekly cisplatin is questionable. ${ }^{27,28}$ One explanation of why we did not observe increased gastrointestinal toxicity with cisplatin is that this occurs less commonly in the intensitymodulated RT era. However, it is also possible that some of the increase in hypovolemia associated with cisplatin was caused by clinically significant diarrhea that was not fully captured in the Medicare claims, which represents a potential limitation of this study.

A strength of this study is the use of a competing risks analysis, which is necessary to accurately assess cancerspecific outcomes in older patients who are at risk for death from other causes. The primary limitation is the retrospective design and the potential for selection bias and confounding. For example, healthier patients may be more likely to be given cisplatin, who then have increased overall survival regardless of the cancer-specific efficacy of cisplatin. To address this issue, our primary outcome was death from cervical cancer, which depends more on cancer-related factors (such as treatment) and is less sensitive to a patient's underlying health status than the outcome of overall survival, and both the propensity score and multivariable analyses adjusted for comorbidity. Nonetheless, our results should be considered hypothesis-generating. SEER-Medicare also lacks radiation details (dose, fields) and recurrence data.

\section{Conclusions}

Although older women constitute an increasing proportion of cervical cancer diagnoses, they have worse outcomes than younger patients and are frequently undertreated. We showed that women aged $\geq 65$ years with cervical cancer undergoing definitive RT had significantly lower risk of dying of cervical cancer if they were treated with cisplatin, despite competing risks for death in an older population. Our results are supported by prior studies showing that age alone does not predict intolerance of combined modality treatment or increased toxicity. Finally, we found that receiving $\geq 5$ cycles of weekly cisplatin was necessary for benefit. Given the paucity of existing data, our study (although retrospective) suggests that older women benefit from combined modality therapy that is standard of care in younger women.

\section{Acknowledgments}

The authors wish to acknowledge the efforts of the Applied Research Program (NCI); the Office of Research, Development, and Information (Centers for Medicare and Medicaid Services); Information Management Services, Inc.; and the SEER program tumor registries in the creation of the SEER-Medicare database. The interpretation and reporting of these data are the sole responsibility of the authors.

Submitted December 11, 2018; accepted for publication February 25, 2019.

Author contributions: Study concept: Xiang, Kidd. Data curation and analysis: Xiang. Investigation: Xiang, Kidd. Methodology: Xiang, Kidd. Project administration, resources, and supervision: Kidd. Drafting of manuscript: Xiang. Critical revisions: Xiang, Kidd.

Disclosures: The authors have disclosed that they have not received any financial considerations from any person or organization to support the preparation, analysis, results, or discussion of this article.

Correspondence: Elizabeth A. Kidd, MD, Department of Radiation Oncology, Stanford University, 875 Blake Wilbur Drive, Room CC-G220A, Stanford, CA 94304. Email: ekidd@stanford.edu

\section{References}

1. Moore KN, Java JJ, Slaughter KN, et al. Is age a prognostic biomarker for survival among women with locally advanced cervical cancer treated with chemoradiation? An NRG Oncology/Gynecologic Oncology Group ancillary data analysis. Gynecol Oncol 2016;143: 294-301.

2. Rositch AF, Nowak RG, Gravitt PE. Increased age and race-specific incidence of cervical cancer after correction for hysterectomy prevalence in the United States from 2000 to 2009. Cancer 2014;120: 2032-2038.

3. Hammer A, Kahlert J, Rositch A, et al. The temporal and age-dependent pattems of hysterectomy-corrected cervical cancer incidence rates in Denmark: a population-based cohort study. Acta Obstet Gynecol Scand 2017;96:150-157.

4. Castanon A, Landy R, Pesola F, et al. Prediction of cervical cancer incidence in England, UK, up to 2040, under four scenarios: a modelling study. Lancet Public Health 2018;3:e34-43.

5. Nosaka K, Shibata K, Utsumi F, et al. Feasibility and benefit of concurrent chemoradiotherapy for elderly patients with uterine cervical cancer. Tumori 2016;102:600-605. 
6. Skaznik-Wikiel ME, Sukumvanich P, Austin RM, et al. Heavy cervical cancer burden in elderly women: how can we improve the situation? Acta Cytol 2012;56:388-393.

7. Nogueira-Rodrigues A, de Melo AC, Garces AH, et al. Patterns of care and outcome of elderly women diagnosed with cervical cancer in the developing world. Int J Gynecol Cancer 2016;26:1246-1251.

8. Diver EJ, Hinchcliff EM, Gockley AA, et al. Assessment of treatment factors and clinical outcomes in cervical cancer in older women compared to women under 65 years old. J Geriatr Oncol 2018;9:516-519.

9. Sharma C, Deutsch I, Horowitz DP, et al. Patterns of care and treatment outcomes for elderly women with cervical cancer. Cancer 2012;118: 3618-3626.

10. Eggemann $\mathrm{H}$, Ignatov $\mathrm{T}$, Geyken $\mathrm{CH}$, et al. Management of elderly women with cervical cancer. J Cancer Res Clin Oncol 2018;144:961-967.

11. Eifel PJ, Winter $K$, Morris $M$, et al. Pelvic irradiation with concurrent chemotherapy versus pelvic and para-aortic irradiation for high-risk cervical cancer: an update of radiation therapy oncology group trial (RTOG) 90-01. J Clin Oncol 2004;22:872-880.

12. Keys HM, Bundy BN, Stehman FB, et al. Cisplatin, radiation, and adjuvant hysterectomy compared with radiation and adjuvant hysterectomy for bulky stage IB cervical carcinoma. N Engl J Med 1999;340:1154-1161.

13. Chemoradiotherapy for Cervical Cancer Meta-Analysis Collaboration. Reducing uncertainties about the effects of chemoradiotherapy for cervical cancer: a systematic review and meta-analysis of individual patient data from 18 randomized trials. J Clin Oncol 2008;26: 5802-5812.

14. Abu-Rustum NR, Yashar CM, Bean S, et al. NCCN Clinical Practice Guidelines in Oncology: Cervical Cancer. Version 2.2019. Accessed January 1, 2019. To view the most recent version, visit NCCN.org.

15. Park JH, Kim YS, Ahn SD, et al. Concurrent chemoradiotherapy or radiotherapy alone for locally advanced cervical cancer in elderly women. Tumori 2010;96:959-965.

16. Wang W, Hou X, Yan J, et al. Outcome and toxicity of radical radiotherapy or concurrent chemoradiotherapy for elderly cervical cancer women. BMC Cancer 2017;17:510.

17. Xiang M, Holsinger FC, Colevas AD, et al. Survival of patients with head and neck cancer treated with definitive radiotherapy and concurrent cisplatin or concurrent cetuximab: a Surveillance, Epidemiology, and End Results-Medicare analysis. Cancer 2018;124:4486-4494.

18. Reeder-Hayes KE, Meyer AM, Hinton SP, et al. Comparative toxicity and effectiveness of trastuzumab-based chemotherapy regimens in older women with early-stage breast cancer. J Clin Oncol 2017;35: 3298-3305.

19. Xiang $M$, Nguyen PL. Significant association of brachytherapy boost with reduced prostate cancer-specific mortality in contemporary patients with localized, unfavorable-risk prostate cancer. Brachytherapy 2015;14: 773-780.
20. Morris M, Eifel PJ, Lu J, et al. Pelvic radiation with concurrent chemotherapy compared with pelvic and para-aortic radiation for high-risk cervical cancer. N Engl J Med 1999;340:1137-1143.

21. Bonner JA, Harari PM, Giralt J, et al. Radiotherapy plus cetuximab for locally advanced head and neck cancer: 5-year survival data from a phase 3 randomized trial, and relation between cetuximab-induced rash and survival. Lancet Oncol 2010;11:21-28.

22. Pignon J-P, le Maître A, Maillard E, et al. Meta-analysis of chemotherapy in head and neck cancer (MACH-NC): an update on 93 randomised trials and 17,346 patients. Radiother Oncol 2009;92:4-14.

23. Atagi S, Kawahara M, Yokoyama A, et al. Thoracic radiotherapy with or without daily low-dose carboplatin in elderly patients with non-smallcell lung cancer: a randomised, controlled, phase 3 trial by the Japan Clinical Oncology Group (JCOG0301). Lancet Oncol 2012;13:671-678.

24. Mak RH, Hunt D, Shipley WU, et al. Long-term outcomes in patients with muscle-invasive bladder cancer after selective bladder-preserving combined-modality therapy: a pooled analysis of Radiation Therapy Oncology Group protocols 8802, 8903, 9506, 9706, 9906, and 0233 J Clin Oncol 2014;32:3801-3809.

25. de Boer SM, Powell ME, Mileshkin L, et al. Adjuvant chemoradiotherapy versus radiotherapy alone for women with high-risk endometrial cancer (PORTEC-3): final results of an international, open-label, multicentre, randomised, phase 3 trial. Lancet Oncol 2018;19:295-309.

26. Rose PG, Ali S, Watkins E, et al. Long-term follow-up of a randomized tria comparing concurrent single agent cisplatin, cisplatin-based combination chemotherapy, or hydroxyurea during pelvic irradiation for locally advanced cervical cancer: a Gynecologic Oncology Group study. J Clin Oncol 2007;25:2804-2810.

27. Chen $\mathrm{X}$, Zou H, Li H, et al. Weekly versus triweekly cisplatin-based chemotherapy concurrent with radiotherapy in the treatment of cervical cancer: a meta-analysis. Int J Gynecol Cancer 2017;27:344-349.

28. Kim YS, Shin SS, Nam J-H, et al. Prospective randomized comparison of monthly fluorouracil and cisplatin versus weekly cisplatin concurrent with pelvic radiotherapy and high-dose rate brachytherapy for locally advanced cervical cancer. Gynecol Oncol 2008;108:195-200.

29. Peters WA III, Liu PY, Barrett RJ II, et al. Concurrent chemotherapy and pelvic radiation therapy compared with pelvic radiation therapy alone as adjuvant therapy after radical surgery in high-risk early-stage cancer of the cervix. J Clin Oncol 2000;18:1606-1613.

30. Nguyen-Tan PF, Zhang $Q$, Ang KK, et al. Randomized phase III trial to test accelerated versus standard fractionation in combination with concurrent cisplatin for head and neck carcinomas in the Radiation Therapy Oncology Group 0129 trial: long-term report of efficacy and toxicity. J Clin Oncol 2014;32:3858-3866.

31. Rose PG, Bundy BN, Watkins EB, et al. Concurrent cisplatin-based radiotherapy and chemotherapy for locally advanced cervical cancer. N Engl J Med 1999;340:1144-1153. 
Supplemental online content for:

\section{Benefit of Cisplatin With Definitive Radiotherapy in Older Women With Cervical Cancer}

Michael Xiang, MD, PhD, and Elizabeth A. Kidd, MD

J Natl Compr Canc Netw 2019;17(8):969-975

eFigure 1: Cumulative Incidence of Death From Cervical Cancer in Propensity Score-Matched Cohorts

eFigure 2: Distribution of Cisplatin Cycles

eFigure 3: Cumulative Incidence of Death in Women Aged $\geq 75$ Years

eFigure 4: Cumulative Incidence of Death in Node-Negative Disease

eFigure 5: Cumulative Incidence of Death in Women Aged $\geq 75$ Years With Node-Negative Disease

eFigure 6: Cumulative Incidence of Death in Women With FIGO Stage I, Node-Negative Disease

eFigure 7: Cumulative Incidence of Death in Women With FIGO Stage II, Node-Negative Disease

eFigure 8: Cumulative Incidence of Death in Women With FIGO Stage I-IIA, Node-Negative Disease With

Tumors $<4 \mathrm{~cm}$

eTable 1: Procedure Claims Codes

eTable 2: Diagnosis Claims Codes

eTable 3: Patient and Tumor Characteristics in Propensity Score-Matched Cohorts 


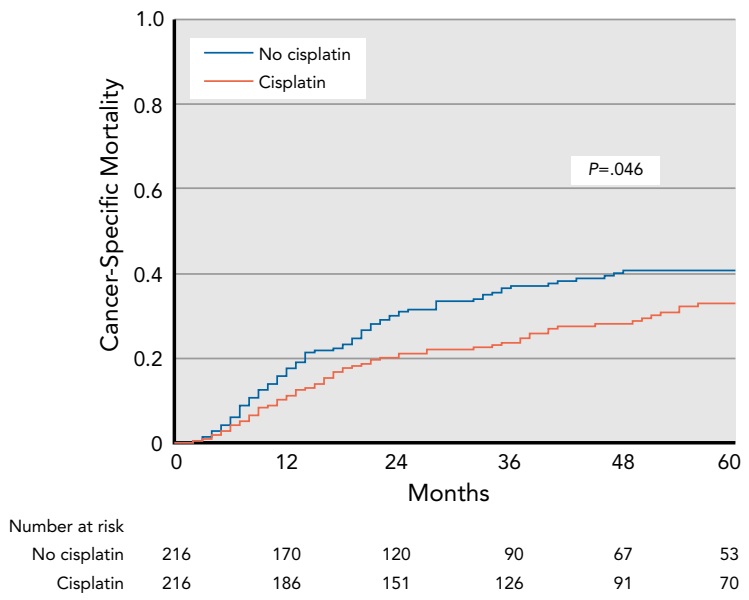

eFigure 1. Cumulative incidence of death from cervical cancer in propensity score-matched cohorts.

A

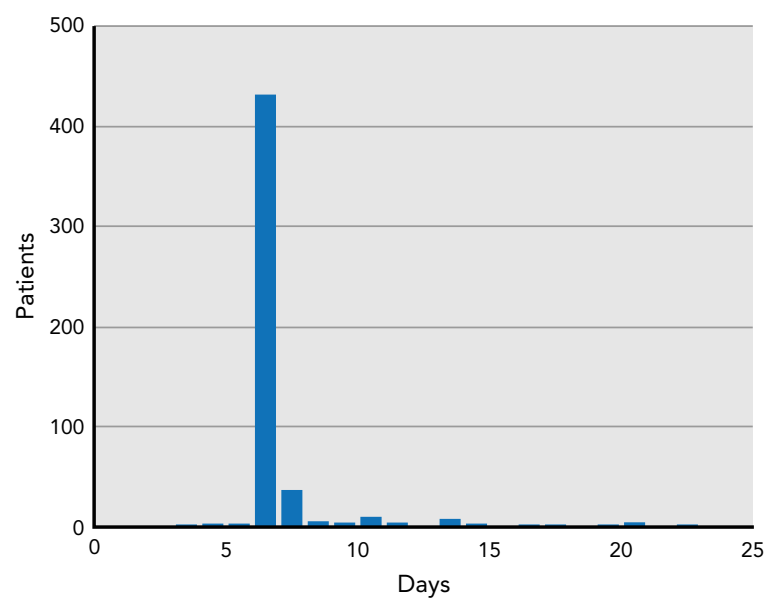

B

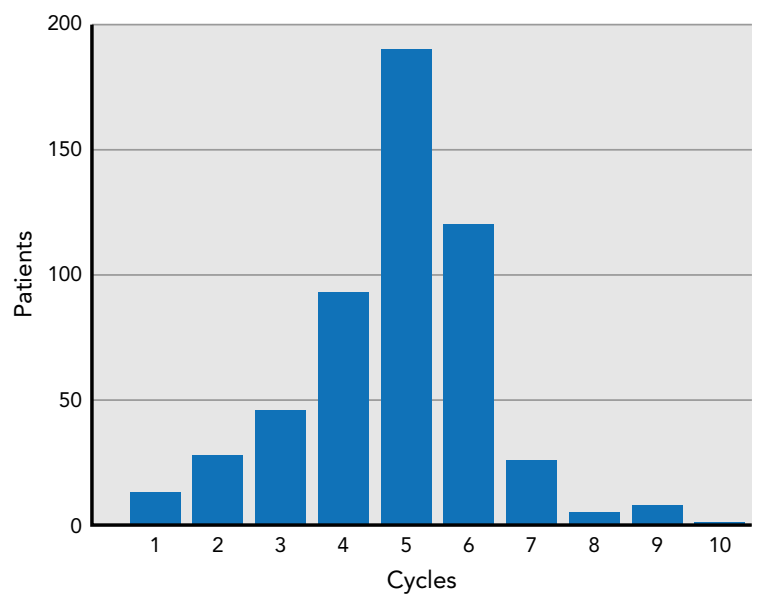

eFigure 2. Distribution of (A) median number of days between consecutive cisplatin cycles in patients who received $>1$ cycle of cisplatin, and (B) number of cisplatin cycles in patients who received cisplatin. 


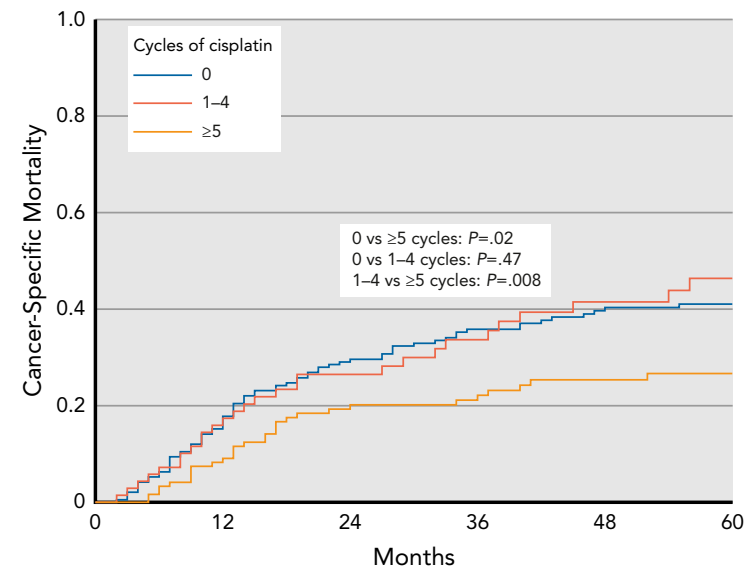

eFigure 3. Cumulative incidence of death from cervical cancer in women aged $\geq 75$ years.

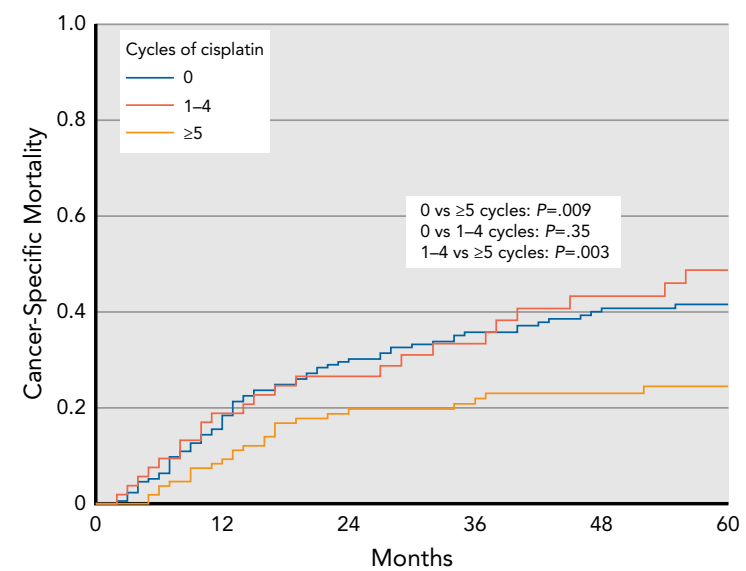

eFigure 5. Cumulative incidence of death from cervical cancer in women aged $\geq 75$ years with node-negative disease.

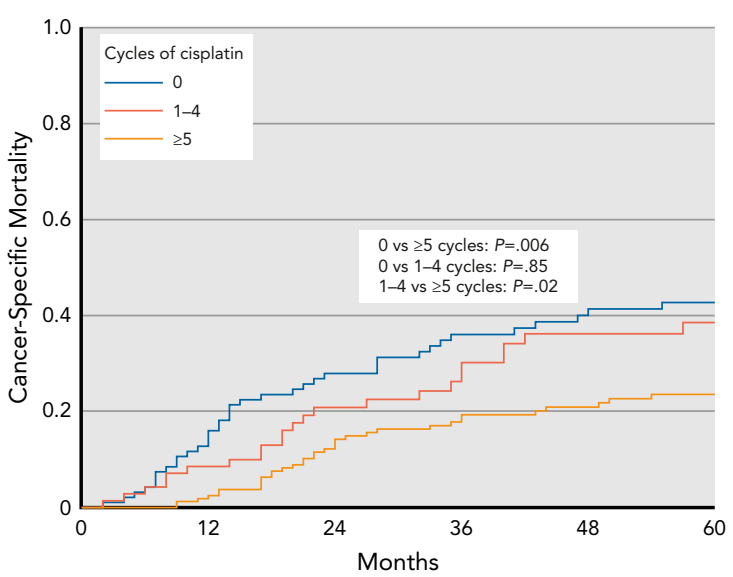

eFigure 7. Cumulative incidence of death from cervical cancer in women with FIGO stage II, node-negative disease.

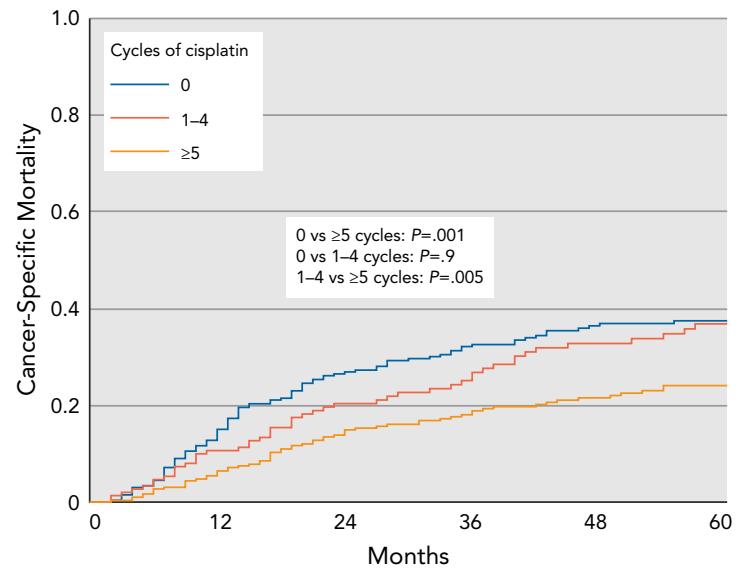

eFigure 4. Cumulative incidence of death from cervical cancer in women with node-negative disease.

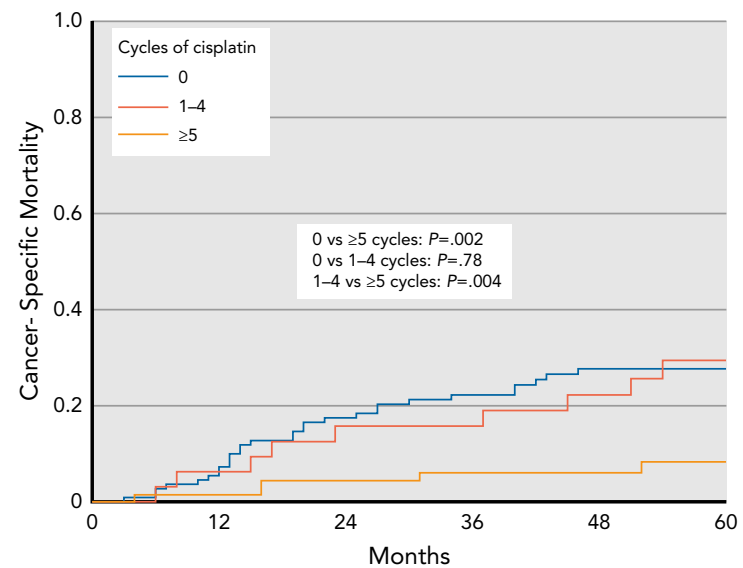

eFigure 6. Cumulative incidence of death from cervical cancer in women with FIGO stage I, node-negative disease.

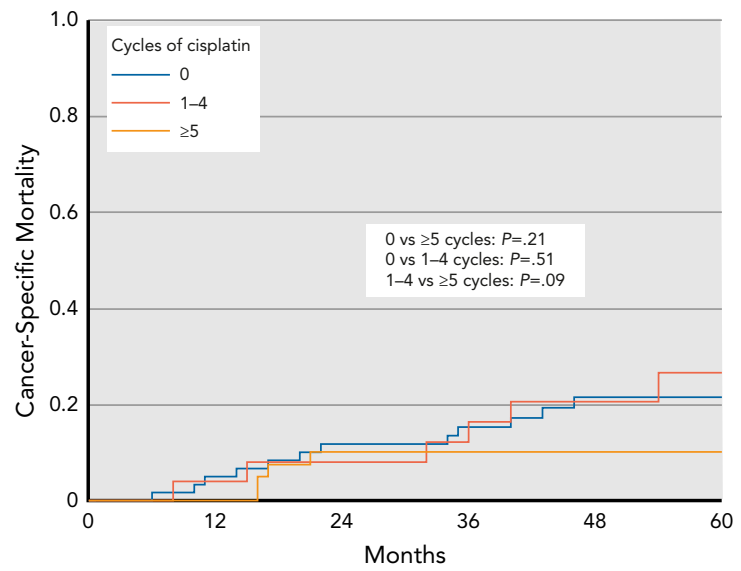

eFigure 8. Cumulative incidence of death from cervical cancer in women with FIGO stage I-IIA, node-negative disease with tumors $<4 \mathrm{~cm}$. 


\section{eTable 1. Procedure Claims Codes}

\begin{tabular}{|c|c|c|}
\hline Procedure & ICD-9 & HCPCS \\
\hline External-beam radiation & $9223,9224,9225,9226$ & $\begin{array}{l}0073 \mathrm{~T}, 4165 \mathrm{~F}, 4181 \mathrm{~F}, 4201 \mathrm{~F}, 77295,77301,77305,77306,77307,77310,77315,77321, \\
\text { 77338, 77380, 77381, 77385, 77386, 77399, 77418, 77520, 77522, 77523, 77525, G0174, } \\
\text { G0178, G6015, G6016 }\end{array}$ \\
\hline Brachytherapy & $9220,9221,9227,9228$ & $\begin{array}{l}55920,57155,57156,58346,77316,77317,77318,77326,77327,77328,77750,77761, \\
77762,77763,77776,77777,77778,77781,77782,77783,77784,77785,77786,77787, \\
\text { 77789, 77790, 77799, C1164, C1174, C1715, C1716, C1717, C1718, C1719, C1720, C1728, } \\
\text { C1796, C1797, C1798, C1799, C1800, C1801, C1802, C1803, C1804, C1805, C1806, C2632, } \\
\text { C2633, C2634, C2635, C2636, C2638, C2639, C2640, C2641, C2642, C2643, C2644, C2676, } \\
\text { C2698, C2699, Q3001, S2270 }\end{array}$ \\
\hline
\end{tabular}

Abbreviation: HCPCS, Healthcare Common Procedure Coding System. 


\section{eTable 2. Diagnosis Claims Codes}

ICD-9 Diagnosis Codes

\begin{tabular}{|c|c|}
\hline Chronic kidney disease & $\begin{array}{l}5820,5821,5822,5824,58281,58289,5829, \\
5830,5831,5832,5834,5836,5837,58381, \\
58389,5839,5851,5852,5853,5854,5855, \\
5856,5859,5880,5881,58881,58889,5889, \\
\text { V420, V4573 }\end{array}$ \\
\hline Diarrhea & 78791 \\
\hline Dysuria & 7881 \\
\hline Cystitis & $5950,59582,5959$ \\
\hline Cytopenia & $28411,2853,28749,2875,28800,28803$ \\
\hline Nausea/Vomiting & $78701,78702,78703$ \\
\hline Hypovolemia & $27650,27651,27652$ \\
\hline
\end{tabular}

\section{eTable 3. Patient and Tumor Characteristics in Propensity Score-Matched Cohorts}

\begin{tabular}{ccc}
$\begin{array}{c}\text { No Cisplatin } \\
(\mathbf{N}=216)\end{array}$ & $\begin{array}{c}\text { Cisplatin } \\
(\mathbf{N}=216)\end{array} \quad P$ Value \\
\hline
\end{tabular}

\begin{tabular}{|lrr}
\hline \multicolumn{3}{l}{ Charlson comorbidity score, $\mathrm{n}(\%)$} \\
\hline 0 & $111(51 \%)$ & $116(54 \%)$ \\
\hline 1 & $49(23 \%)$ & $48(22 \%)$ \\
\hline$\geq 2$ & $56(26 \%)$ & $52(24 \%)$ \\
\hline Median age (SD), y & $76(6.6)$ & $75(5.8)$ \\
Median diagnosis year (SD) & $2008(2.7)$ & $2008(2.8)$ \\
\hline Race, $\mathrm{n}(\%)$ & & \\
\hline White & $150(69 \%)$ & $144(67 \%)$ \\
\hline Nonwhite & $66(31 \%)$ & $72(33 \%)$ \\
Region, $\mathrm{n}(\%)$ & & \\
\hline Northeast & $49(23 \%)$ & $52(24 \%)$ \\
\hline West & $16(7 \%)$ & $12(6 \%)$ \\
\hline Midwest & $30(14 \%)$ & $34(16 \%)$ \\
\hline South & $121(56 \%)$ & $118(55 \%)$ \\
\hline
\end{tabular}

Marital status, $\mathrm{n}(\%)$

\begin{tabular}{lrr} 
Married & $60(28 \%)$ & $47(22 \%)$ \\
\hline Other & $156(72 \%)$ & $169(78 \%)$
\end{tabular}

Census tract poverty level, $\mathrm{n}(\%)$

\begin{tabular}{lll}
\hline$<10 \%$ & $77(36 \%)$ & $81(38 \%)$ \\
\hline $10 \%-20 \%$ & $76(35 \%)$ & $76(35 \%)$ \\
\hline$>20 \%$ & $63(29 \%)$ & $59(27 \%)$
\end{tabular}

Urban/Rural residence, $\mathrm{n}(\%)$ .87

\begin{tabular}{lrr}
\hline Big metropolitan & $118(55 \%)$ & $120(56 \%)$ \\
\hline Other & $98(45 \%)$ & $96(44 \%)$
\end{tabular}

T stage (FIGO), n (\%)

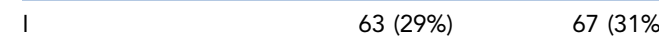

II $94(44 \%) \quad 88(41 \%)$

III-IVA $59(27 \%) \quad 61(28 \%)$

$\mathrm{N}$ stage, $\mathrm{n}(\%)$

\begin{tabular}{rrr} 
N0 & $190(88 \%)$ & $191(88 \%)$ \\
\hline N1 & $26(12 \%)$ & $25(12 \%)$
\end{tabular}

Histology, n (\%)

Squamous cell $\quad 178(82 \%) \quad 174(81 \%)$

carcinoma

Adenocarcinoma $\quad 38(18 \%) \quad 42(19 \%)$

Chronic kidney disease, $\mathrm{n}(\%)$

\begin{tabular}{lrr}
\hline No & $190(88 \%)$ & $187(87 \%)$ \\
\hline Yes & $26(12 \%)$ & $29(13 \%)$
\end{tabular}

